\title{
LOS CUIDADOS DE ENFERMERÍA EN LA INFANCIA: «EL HOSPITAL DEL NIÑO JESÚS»
}

\author{
Ma Francisca Casas Martínez \\ Teresa Ruiz Ureña \\ Begoña Carbelo Baquero \\ Profesoras Titulares de Enfermería \\ Teresa Miralles Sangro \\ MARTA Romero LLORT \\ Profesoras Asociadas de Enfermería
}

Escuela de Enfermería y Fisioterapia

de la Universidad de Alcalá. Alcalá de Henares. Madrid

\author{
Correspondencia: \\ $\mathrm{M}^{\mathrm{a}}$ Francisca Casas Martínez \\ Departamento de Enfermería y Fisioterapia. \\ Universidad de Alcalá \\ Campus Universitario S/N \\ E-28871 Alcalá de Henares (Madrid)
}

\section{RESUMEN}

En el presente trabajo se analiza el momento histórico en el que se funda el Hospital Pediátrico del Niño Jesús de Madrid, así como la evolución que ha sufrido la Atención Enfermera a la Infancia.

\section{NURSING CARE FOR INFANTS: «EL HOSPITAL DEL NIÑO JESÚS»}

\section{ABSTRACT}

The historical scenario in which the Hospital Pediátrico del Niño Jesús was created is analyzed in the present paper, as well as the evolution undertaken in nursing attention to infants.

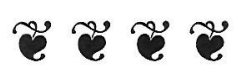

\section{INTRODUCCIÓN}

Históricamente la atención al niño, es poco conocida y ha estado estrechamente ligada a la propia consideración que la sociedad ha tenido de la infancia en los diferentes momentos históricos.

Aunque los niños, los hijos siempre han sido un valor importantísimo en todas las culturas, de manera que determinaban también el valor de la mujer (la que no podía tener hijos era absolutamente desvalorizada y acaso lo sigue siendo también en nuestra cultura), sin embargo podían convivir situaciones de reverencia y búsqueda de herederos, con otras en que no se podían controlar el nacimiento en exceso y los niños sufrían el descuido y el abando- no. Las altas tasas de natalidad han coincidido con altas tasas de mortalidad infantil.

El gran cambio de atención a la infancia se produce cuando se desarrolla la vida en la ciudad, aumenta la población y la abundancia de hijos ya no es una bendición de Dios y brazos para trabajar en el campo, si no bocas para comer.

El abandono de niños en los conventos, hospitales, casas de caridad ha sido una constante en la Historia y se recoge en diversos textos. En 1198, Inocencio III preocupado por el gran número de cuerpos de niños que aparecía en el Tíbet, fundó el hospital de Santa María de Massía, para niños abandonados. 
Eseverri (1985)', recoger también este hecho en Siglo XIII en Barcelona.

El nacimiento de las Hijas de la Caridad (1670), se liga a la atención a los niños abandonados y enfermos.

La Santa Creu de Barcelona, recoge en sus reglamentos de 1756 la figura de personas encargadas de cuidar a los niños abandonados, «Las mares de les Dides (lactantes), del Govern (niños) l de las Doncelles (niñas y jóvenes) ${ }^{2}$.

Los niños abandonados se recogen en casas de misericordia, asilos, hospicios. Estas instituciones constituyen un $41 \%$ frente a un $13 \%$ de Hospitales en el siglo XVIII. El transito del hospital medieval, en el que en sus orígenes se albergaban problemas sociales, hacia una filosofía sanitaria con dedicación mayor a la curación de enfermos, afectará también a la infancia, dando lugar en el siglo XIX a la separación del niño de los adultos y al nacimiento de hospitales de niños.

\section{LAS FUENTES}

La fuentes documentales utilizadas para el estudio, además de las citadas al final del documento, fueron algunos libros de registros de ingresos que posteriormente han desaparecido. Fotografías y los relatos de vida de personas que llevan gran parte de este siglo dedicados al hospital

\section{EL CONTEXTO SOCIAL E HISTÓRICO EN LA FUNDACIÓN DEL HOSPITAL}

Todas estas figuras y situaciones responden a la demanda de la sociedad: No existía el control de la natalidad y las condiciones higiénicas eran lamentables. Los niños que sobraban eran abandonados.

Si la mortalidad infantil era alta en general, las posibilidades de supervivencia de estos niños aban- donados era mínima. Las inclusas eran lugares en los que los niños morían. Desgraciadamente estas escenas las podemos conocer con el mismo realismo en la actualidad, recordemos los hospitales de la muerte en China (El País 1995)

La industrialización y el desarrollo de las ciudades sólo hizo que agravar este problema.

Las primeras instituciones e intentos de sacar a los niños de los hospitales de adultos se sitúa en París con la fundación de «La Maison de L'Enfant Jesús» fundado en 1751 , y posteriormente Hôpital des Enfants Malades que serviría de modelo para la fundación del Hospital del Niño Jesús fundado en 1879 en Madrid.

Cuando se fundó este hospital, las tasas de mortalidad infantil en Madrid eran de las mas elevadas de Europa, estando entre un 26'7 por mil y un 37'9 por mil ${ }^{3}$. Según algunos autores esta situación demográfica característica del Antiguo Régimen ${ }^{4}$, se debe a enfermedades sociales, potenciadas por las deficiencias higiénicas, lo que originaba un gran número de enfermedades infecciosas.

Nuestro país permanecía muy atrasado en la legislación que trataba de ordenar la mejora de la salud pública, como ejemplo tenemos que en Inglaterra se publica en 1874 el Estatuto de Salud Pública y en España aparece su equivalente El Estatuto $\mathrm{Mu}-$ nicipal en 1924, fruto del Movimiento Reformista y del que nació otra importante institución como fue la Escuela Nacional de Puericultura (1923), adscrita al Consejo Superior de Protección de la Infancia. (de acuerdo con la Ley de Protección de la Infancia de 1904 y el Reglamento de Puericultura de 1910).

Los nuevos valores son difíciles de aceptar por la moralidad tradicional de la sociedad española, tan acostumbrada a asumir la caridad como un deber, planteando una dicotomía entre el Estado, últi-

1. ESEVERRI CHAVERRI, C.: (1995) Historia de la Enfermería Española. Universitas. Madrid.

2. FARGUES GARCÍA, I y TEY FREISA, R.: Enfermería en el siglo XVIII. El Hospital de Santa Creu de Barcelona. Rol de Enfermería no 222. Febrero 1997:77-83

3. GÓMEZ REDONDO: El descenso de la mostalidad Infantil en Madrid. 1900-1979. Revista Española de Investigaciones Sociológicas, $\mathrm{n}^{\circ}$ 32. Octubre-Diciembre 1985.

4. FERNÁNDEZ FERRÍN, C, et al: (1994) Enfermería Fundamental. Masson, SA. Madrid.

5. SILES GONZÁLEZ,J: (1996) Pasado, presente y futuro de la Enfermería en España. Consejo de Enfermería de la Comunidad de Valencia. Valencia. 
mo responsable de un sistema de beneficencia pública del país, y la Iglesia, defensora de la moral tradicional y de la caridad en la labor asistencial. En la nueva situación que surgió a finales del siglo pasado la Iglesia se encargó de los sectores benéfico-asistenciales: orfanatos, casas de expósitos, asilos. Y el estado y los médicos se responsabilizaron de los Hospitales.

\section{LA FUNDACIÓN DEL HOSPITAL NIÑO JESÚS}

En este contexto social y político la Duquesa de Santoña funda en 1879 el Hospital del Niño Jesús, como decíamos, a semejanza del Hôpital des Enfants Malades de París, fundado un poco antes, que sin duda conocía y que le sirvió de modelo, con el objetivo de sacar a los niños enfermos de los hospitales de adultos.

Propiciado por la Real Orden de 1876 que autoriza la fundación de hospitales de niños en Madrid, se constituye la «Asociación Nacional para la Fundación y Sostenimiento de Hospitales de Niños en España», el primero de ellos sería el del Niño Jesús.

En un principio el hospital estuvo en la calle Laurel $n^{\circ} 23$, barrios del Gasómetro y las Peñuelas, distrito de la Inclusa, barrios marginales, lo que justifica también el gran índice de mortalidad que se daba en ese momento en estos barrios en concreto, que era la mas alta de Europa.

Posteriormente pasó al lugar que ocupa en la actualidad, en la calle Menéndez Pelayo, al lado del Parque del Retiro y de la Estación de Ferrocarril, ya en el campo. En su fundación poseía cuatro salas y la capilla que pronto fueron creciendo hasta configurarse como es en la actualidad en la epidemia de polio del 56.

En 1889, pasó a ser administrado por la Excelentísima Junta Provincial de Beneficencia, tras algunos problemas habidos entre la fundadora y los facultativos, que buscaban sin duda el disponer de mayor autonomía en el desarrollo de sus funciones.

\section{EL PERSONAL}

Es una fundación particular, en la que siguen presentes los valores de la caridad, pero con un claro interés científico, pues buscó los mejores médicos (en 1888 se funda la primera Cátedra de Pediatría en la Universidad Española, lo que nos hace pensar en un movimiento general que orienta la formación de los médicos y las corrientes sociales hacia la especial atención de los niños). Así mismo se busca personal de enfermería especializado para que se ocupe de los niños en su fundación, y las mejores profesionales de enfermería que encontró en ese momento la fundadora fueron La Hijas de la Caridad, con las que firmó un convenio el 1 de junio de 1878, estando presentes la propia Duquesa, el Vicario General de la Congregación D. Antonio Fiah y la Visitadora Sor Julia Kieffer. La primera superiora se llamaba Sor Rosalía Blanchard ${ }^{6}$.

Como es típico en todos los contratos que firmaban las Hijas de la Caridad en su primer artículo se recoge que las $\mathrm{H}$. de $\mathrm{C}$. tienen sus reglas que no se las pueden cambiar y sólo dependen de los Superiores de su Comunidad. Y la Superiora sólo daba cuentas de su hacer a la presidenta del patronato. El resto de los empleados del hospital dependen de la Madre Superiora excepto los Señores Facultativos y Eclesiásticos. También destacamos el Art. 14 que recoge que «no se cuidarán de nada fuera del hospital, ni a las mujeres de mal vivir, ni a las que padezcan enfermedades que procedan de ello, ni asistan a las que estén de parto».

En una nómina de personal de diciembre de 1887 destaca que había 5 enfermeras que cobraban 15 pts.

Este nombre es evidente que no correspondía a la enfermera actual, podemos decir como Doña Concepción Arenal (1870:51-55) escribía en su detallado análisis del Hospital General de Madrid, en el que relata que las enfermeras laicas carecían de los mínimos conocimientos del arte de curar? Sólo

6. Información facilitada por Las Hijas de la Caridad, que residen en un pequeño apartamento de la que fue antigua residencia de la monjas y que poseen dicho convenio.

7. ARENAL, C.: (1894) Obras Completas. Tomo I y II. Librería Visctoriano Suarez. Madrid. Citado en Parte V, capítulo 17 por Hernández Rodríguez, Lasante Calderay y Siles Rodríguez: «El Movimiento Reformista durante el siglo XIX: Su influencia en la Enfermería Española». Síntesis. Madrid. :227-230. 
cobraban menos que ellas la encargada de pasillos (15 pts.) la cocinera (9 pts,) las lavanderas, la encargada del ropero. Los dos mozos y la portera, estaban mejor considerados que ellas y cobraban 20 pesetas, igual que las Hermanas de la Caridad. Estas cifras son similares a las aportadas por el profesor Siles González (1996) en «El sistema de beneficencia y la Enfermería Decimonónica. El caso de Alicante». ${ }^{8}$

Confirma el que esas enfermeras deberían corresponder a personas de servicio el relato recogido por Ollero Caprani (1980); con motivo de un desgraciado accidente ocurrido a la niña Elvira Morellon y González en la sala de Santa Lucía, que había ingresado por un problema oftalmológico y que falleció de graves quemaduras. A raíz de este accidente se recomienda "Que las enfermeras de las salas además de reunir condiciones para su desempeño, tengan entre 18 y 35 años, y que cesen las que no tengan esa edad y se ocupen las Hermanas de las Enfermerías y mayor vigilancia y cuidados de los enfermos».

Carecemos de noticias de este periodo hasta que en el 27 de noviembre de 1889 pasa a ser administrado por la Excelentísima Junta Provincial de Beneficencia.

\section{ESTRUCTURA Y ORGANIZACIÓN}

Desconocemos como estaba estructurada la atención de enfermería en el hospital en la época de su fundación. Se han perdido los archivos y si existen otros documentos no hemos podido acceder a ellos.

Disponemos del testimonio principalmente de una Hermana de la Caridad Sor Mercedes, que tiene en la actualidad 90 años y que lleva en el Hospital desde 1942. A través de su relato podemos reconstruir una larga época de la historia.

Sor Mercedes estudio enfermería en la Cruz Roja de Melilla y trabajó en el Hospital de San Carlos de Atocha. Durante la guerra civil estuvo en un pequeño Hospital de Sangre, en la Tunara, en La Línea de la Concepción (Cádiz) y tras una corta es- tancia en Marsella, pasó al Hospital del Sagrado Corazón de Barcelona. Desde allí llegó a este hospital, en el que lleva 55 años. Ella siempre se ha identificado con la atención a los niños.

El hospital siempre ha estado estructurado en Salas. Cada una de ellas era un mundo bastante cerrado dirigido por un jefe Clínico y una Hija de la Caridad, la enfermera. El nombre de la sala era de algún Santo, bajo cuya advocación estaban los niños enfermos, por ejemplo Santa Lucía, para la sala de los niños con problemas en la vista. Otros santos y santas se elegía para proteger a los niños ellos y para proteger a las niñas ellas como El Santo Angel, Santa Mónica, Santiago, San Ildefonso... etc.

Estas salas como decíamos tenían bastante independencia, con su propia ropa, perfectamente marcada, su material y su personal. Había cierta rivalidad entre ellas, buscando para cada sala algo que la identificara y que diera mas importancia al personal de ella.

\section{ATENCIÓN DE ENFERMERÍA}

Los cuidados enfermeros se basaban en atender las necesidades básicas, la alimentación, la higiene y la seguridad. También los aspectos espirituales .

No existían registros de enfermería, salvo un libro de ingresos de los que quedan algunos que permitieron recuperar datos de la gran epidemia de polio del año 1956, en la que este hospital tuvo un gran protagonismo.

Los niños que padecían la poliomielitis eran remitidos de toda España y de sus colonias. Llegaron a funcionar 20 pulmones de acero, que era la alternativa cuando en el proceso de la enfermedad se producía la parálisis de los músculos respiratorios. (Incluso se hizo uno a medida que incluyera sólo el trono y que permitiera llevar a un niño a Lourdes)

El único tratamiento que se les administraba era vitamina B y el resto eran cuidados de enfermería. Para los dolores musculares de les aplicaba a todos los niños «fomentos calientes» que consistía en colocar por toda la zona afectada compresas humedecidas en agua muy caliente. Sor Mercedes nos rela- 
taba como se pasaba el día poniendo las compresas a los niños. Terminaba con el último de sus pacientes y ya tenía que volver a empezar.

Recuerda la epidemia como un periodo de mucho trabajo. Tenían el Hospital lleno. (llegaron a tener 600......niños ingresados). Había 20 Hermanas trabajando y para que pudieran descansar el domingo venían Hermanas de las que se dedicaban a la enseñanza a cuidar de los niños.

La familia era apartada de los niños, y no se contaba con ella para nada. La característica que decíamos de que fueron enviados niños de toda España propició que algunos fueran «olvidados» por sus padres, quizás debido a la distancia y a la penuria económica que no permitía hacer viajes para visitar al hijo. Máxime cuando sabían que sus hijos estaban incluso mejor que en su casa. Se desarrolló mucho la unidad de Traumatología y Ortopedia Infantil, así como la Fisioterapia, ayudas claves para la recuperación de los niños.

Como anécdota recuerda que al querer dejar en Guinea a dos hermanos con sus padres, nadie quiso acogerlos y tuvieron que volverlos a traer al hospital y buscar otra solución.

También se abrió un colegio para que los niños iniciaran o siguieran su formación escolar.

Permanecía presente la actitud de caridad. Acudían chicas de las que estaban realizando el «Servicio Social» a cuidar a los niños, con un claro matiz de caridad, que seguía impregnando la asistencia.

\section{ETAPA ACTUAL}

En 1960 se abre en Madrid la Ciudad Sanitaria La Paz con un Hospital Infantil de la Seguridad Social. Esto supuso la decadencia del «Hospitalllo», como era llamado. En esta etapa el hospital depende de la administración local, tiene escasez de recursos y claramente se queda trasnochado.

Posteriormente, en 1976 la Seguridad Social, establece un convenio con el Hospital del niño Jesús, integrándolo en su red de hospitales como hospital Nacional y dotándolo de recursos que permitieran que se incorporara a la nueva situación social y científica. Como consecuencia de dicho convenio permanece todo el personal que existía y se incor- pora un numeroso grupo de médicos y enfermeras. Todos venían en general de hospitales muy modernos y tenían muchas ganas de trabajar y cambiar el mundo asistencial. No en vano estamos hablando de 1976, momento de profundo cambio social. Recordemos que también se estrenaba la democracia.

Se encuentran con un hospital con una estructura en apariencia congelada e idéntica a la de su fundación. Seguía ordenada en salas, con su jefe clínico y su supervisora que en muchos casos era una Hija de la Caridad.

La documentación que existía era un libro de ingresos, la hoja de temperaturas y las órdenes del médico.

Era muy distinta una la sala de otras. En la de las monjas, estas tenían la llave y pasaban visita. Las salas con supervisora laica solían corresponder también a actitudes mas permisivas.

El hospital tenía una gran ocupación y destacaban los niños que ingresaban con deshidrataciones de hasta $3^{\circ}$ grado. (Recordemos que en ese momento no existía la Atención Primaria y había muy pocos pediatras que debían atender a una gran población infantil en los barrios periféricos).

De esta primera época lo que mas se recuerda era el grave problema que suponía el separar a los niños de sus padres, en algunos casos peor enfermedad que la que les había llevado al hospital. Había que arrancar literalmente al niño de los brazos de su madre. Algunos padres venían a ver a sus hijos y se refugiaban detrás de un periódico para que el niño no les viera y no tuvieran que sufrir de nuevo la separación.

Vivimos los grandes cambios sociales y políticos y en 1983 cuando se publica «El Nuevo Modelo de gestión Hospitalaria» se produce un cambio en la Dirección de enfermería. Este cambio supuso también un cambio profundo en la ideología del cuidado enfermero.

La primera revolución fue propiciada por el «Plan de Humanización de la Asistencia». Este plan dio pie a permitir la permanencia de las madres y padres con sus hijos. Todas las enfermeras apoyaron y defendieron este derecho fundamental de los niños que ya se había promulgado por la Asamblea 
General de las Naciones Unidas en 1959 y que en 1979 coincidiendo con el aniversario y aprovechando que era el Año Internacional del Niño, seguía teniendo que ser recordado porque aún no se respetaba.

Las enfermeras se erigieron en verdaderas defensoras de este derecho. Había muchas cosas en contra, principalmente la inercia de muchos años de cuidar de otra manera y el rechazo y temor al cambio que siempre está presente. Sin embargo pronto se pudo llevar a cabo y el hospital se erigió de nuevo pionero en esta forma de cuidar a los niños. Paralelo a esta revolución se fueron creando nuevos registros que respondían a otra forma de entender la atención enfermera?

Una nueva ideología se comenzaba a fraguar. Las Salas seguían con su nombre de santo o santa, pero además los niños se ingresaban por edades y también, como en todas partes por especialidad médica. Ya no se separaban los niños de las niñas.

Cada sala dejó de ser un mundo cerrado, se hizo una unidad de dietética que aseguraba mejor la calidad de las dietas, una Farmacia con unidosis que racionaliza el uso de medicamentos y el gasto. ya no hacía falta tener la ropa marcada, había recursos para todos.

Cada sala podía competir en las sesiones de enfermería en las que se debatían los diagnósticos enfermeros mas empleados.

Se desecharon los uniformes blancos, que los niños asocian con dolor y se procedió también a una remodelación del antiguo edificio, rescatando lo histórico y utilizando los recursos mas modernos y adecuados a la edad de los niños.

La humanización de la asistencia dejó de ser el objetivo pues el objetivo era la atención integral.
Nadie cuestionaba la presencia de la familia pues, acaso se puede cuidar a un niño sin ella?

\section{CONCLUSIONES}

Podemos decir que el hospital del Niño Jesús de Madrid, forma parte del paisaje de esta cuidad y en ella es conocido por la mayoría de sus habitantes.

Es el hospital de referencia al que acuden numerosos habitantes de Madrid, precisamente porque les da confianza y también porque siempre son bien acogidos.

La enfermería que ha cuidado a los niños en este hospital ha contribuido especialmente al desarrollo de los cuidados enfermeros a la infancia y actualmente trabaja en la validación de diagnósticos enfermeros, colaborando así al desarrollo de la profesión .

Recuperar su historia nos permite reconocerla e impedir que se olvide.

\section{BIBLIOGRAFÍA}

HERNÁNDEZ CONESA, J.: (1994) Historia de la Enfermería.Madrid. McGraw-Hill Interamericana

JIMÉNEZ SERRANO, C.: «La oftalmología en el Hospital del Niño Jesús de Madrid. Análisis aistencial. (1893-1940)». Tesis Doctoral. Director Pedro Navarro. 1994. Universidad Complutense de Madrid. Facultad de Medicina. Departamento de Salud Pública e Historia de la Ciencia.

OLLERO CAPRANI, J.M.: «El Hospital del Niño Jesús de Madrid. (1877-1919)». Tesis Doctoral. Director Pedro Navarro. 1990. Universidad Complutense de Madrid. Facultad de Medicina. Departamento de Salud Pública e Historia de la Ciencia.

SANTO TOMÁS PÉREZ, M et al.: (1995) Enfermería Fundamental. Barcelona. Masson-Salvat.

9. Deseamos destacar la labor llevada a cabo en este sentido por la Dirección de Enfermería y especialmente por la Adjunta de Formación Continuada Doña Henar Sastre Domingo que lideró el cambio en la atención enfermera a la infancia. 